\title{
Tratamiento de los aceites vegetales usados, como materia prima para la elaboración de biodiésel en la ciudad de Arequipa
}

\author{
Treatment of used vegetal oils, like raw material for the elaboration \\ of biodiesel in the city of Arequipa \\ Rosa María Sotomayor Zúñiga*, \\ Hortencia Elena Huaco Álamo**, Rafael Cuentas Machicao***
}

http://dx.doi.org/10.21503/CienciayDesarrollo.2006.v7.06

\section{RESUMEN}

Las hipótesis de la investigación son: a) Se puede obtener combustible ecológico (biodiésel) a partir de aceites usados, mediante la transesterificación, usando alcohol etílico, y b) Se puede disminuir el grado de contaminación de las aguas efluentes de los principales restaurantes de Arequipa, mediante el tratamiento de los aceites usados que se generan y a través de asesoramiento técnico correspondiente en la separación de este desecho.

Para la investigación se tomaron en cuenta las siguientes variables: a)Variables dependientes: calidad del biodiesel, punto de inflamación, \% de agua y sedimentos, temperatura de destilación, gravedad API, y b) Variables independientes: \% de agua y sedimentos de los aceites usados, $\mathrm{pH}$ de aceites usados, temperatura de la materia prima en el proceso, temperatura de la reacción, razón alcohol/aceite, concentración y tipo de catalizador, RPM de agitador.

Palabras clave: biodiésel, combustible ecológico.

\section{ABSTRACT}

The Hypotheses of the investigation are: a)Ecological fuel (biodiesel) from oils used by means of the transesterificación can be obtained using ethylic alcohol, and b) The degree of contamination of waters can be fallen effluents of the main restaurants of Arequipa, by means of a treatment to the used oils that are generated, and through corresponding technical advising in the separation of this remainder.

For the investigation take into account the following variables: a)Dependent variable: Quality of the Biodiesel, Flash point; \% Water and sediments; Temperature of distillation; Gravity API, and b) Independent Variables: \% Water and sediments of used oils, used oil $\mathrm{pH}$, Temperature of the raw material in the process, Temperature of the reaction, Reason of the alcohol to oil, Concentration and type of catalyst, RPM of agitater.

Key words: biodiesel, ecological fuel.

\footnotetext{
* Docente de la Universidad Alas Peruanas, filial Arequipa.

"Estudiante de Ingeniería Ambiental de la Universidad Alas Peruanas, filial Arequipa.

".* Estudiante de Ingeniería Ambiental de la Universidad Alas Peruanas, filial Arequipa.
} 


\section{INTRODUCCIÓN}

Actualmente, la posible extinción a mediano plazo del aprovisionamiento de las reservas fósiles, la mayor incidencia de una conciencia ambientalista y la realidad concreta del deterioro del medio ambiente han modificado la situación precedente y reactivado la búsqueda de combustibles más amigables con nuestro medio.

El aceite de freír que desechamos en casa tiene como destino habitual el desagüe de la cocina o el inodoro. Con el tratamiento adecuado, todo ese aceite desperdiciado podría convertirse en jabón, fertilizantes y hasta combustible con el que alimentar los vehículos diésel.

Toda industria gastronómica tiene efluentes líquido con contendido de aceite cuya calidad puede variar dependiendo del tipo de servicio que presten. Los aceites vegetales usados resultan una fuente de energía renovable y limpia, los mismos que adecuadamente utilizados pueden contribuir a la conservación del medio ambiente, gracias al reciclado de productos de desecho como los que origina la industria gastronómica.

La justificación para esta investigación, desde el punto de vista tecnológico, es la disminución de emisiones contaminantes de vehículos para reducir los gases invernadero producidos por el transporte.

Desde el punto de vista social, la justificación es proveer de una alternativa de utilización de las tierras agrícolas dedicadas al cultivo de materias primas para la elaboración de aceites vegetales, tierras que, por razones de mercado, están siendo abandonadas por los agricultores.

Como justificación general podemos plantear la reutilización de efluentes líquidos con contendido de aceite de la industria gastronómica.

Los objetivos planteados son: a)evaluar la producción de biodiésel a partir de aceites usados con alcohol etílico, b)proponer modelos tecnológicos para producción de biodiésel a pequeña escala, y c)evaluar el funcionamiento y las emisiones de biodiésel en motores y generadores diésel.

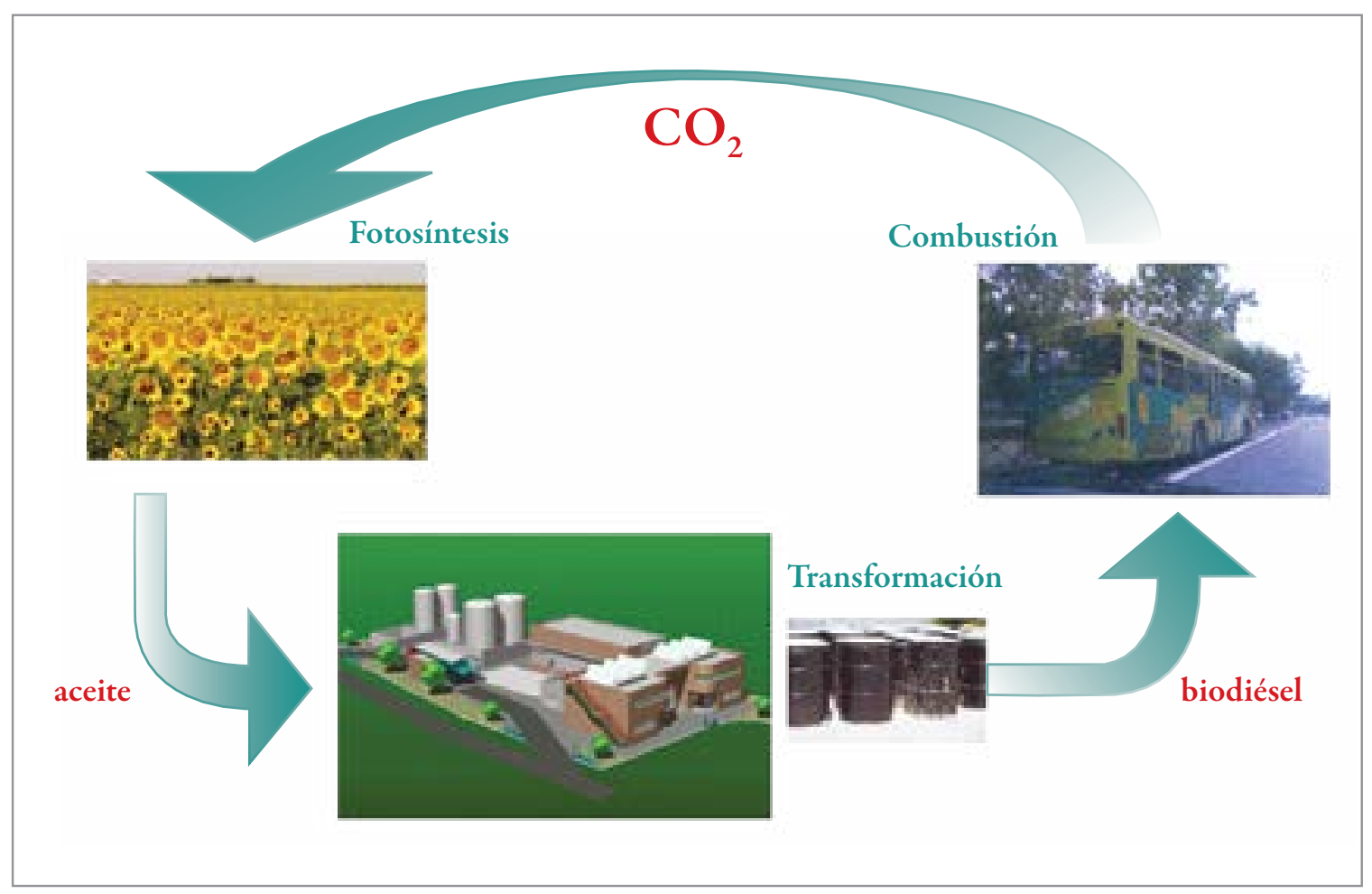




\section{MATERIAL Y MÉTODOS}

El biodiésel tiene como materia prima semillas aceitosas como las de mostaza, maíz, sorgo, soya, palma africana, girasol, cacahuate y coco, además de la grasa animal y los aceites usados de cocina.

\section{Transesterificación del aceite}

El biodiésel es un gasóleo que se obtiene por la transesterificación de triglicéridos (aceite). El producto obtenido es muy similar al gasóleo obtenido del petróleo (petrodiésel), y puede usarse en motores de ciclo diésel, aunque algunos motores requieren modificaciones.<smiles>[R]C(=O)OC[C@@H](O)[C@H](O)[C@]([R])(CO)[C@H](O)CO</smiles>

El proceso de transesterificación consiste en combinar el aceite (normalmente aceite vegetal) con un alcohol ligero, normalmente etanol, lo que deja como residuo glicerina, que puede ser aprovechada por la industria cosmética, entre otras. Se justifica realizar la transesterificación del aceite vegetal y de la grasa animal porque hace posible conseguir valores de viscosidad con el metil éster o etil éster dentro del margen que especifica el petrodiésel (N2-S15, $1.9-4.1 \mathrm{~mm}^{2} / \mathrm{S}$ at $40 \mathrm{C}$. Sin embargo, es importante indicar que aceites de cocinar y vegetales usados (colza, soya y de maní), luego de ser sometidos a proceso de limpieza, se han usado como combustibles directamente en el motor diésel sin aplicarle el proceso básico de transesterificación.

\section{Materiales}

- Aceite usado.

- Hidróxido de sodio (o de potasio).

- Alcohol etílico o metílico.

- Birreactor Fed Batch.

- Condensador.

- Agitador.

- Tanques de lavado.

- Peras de decantación.

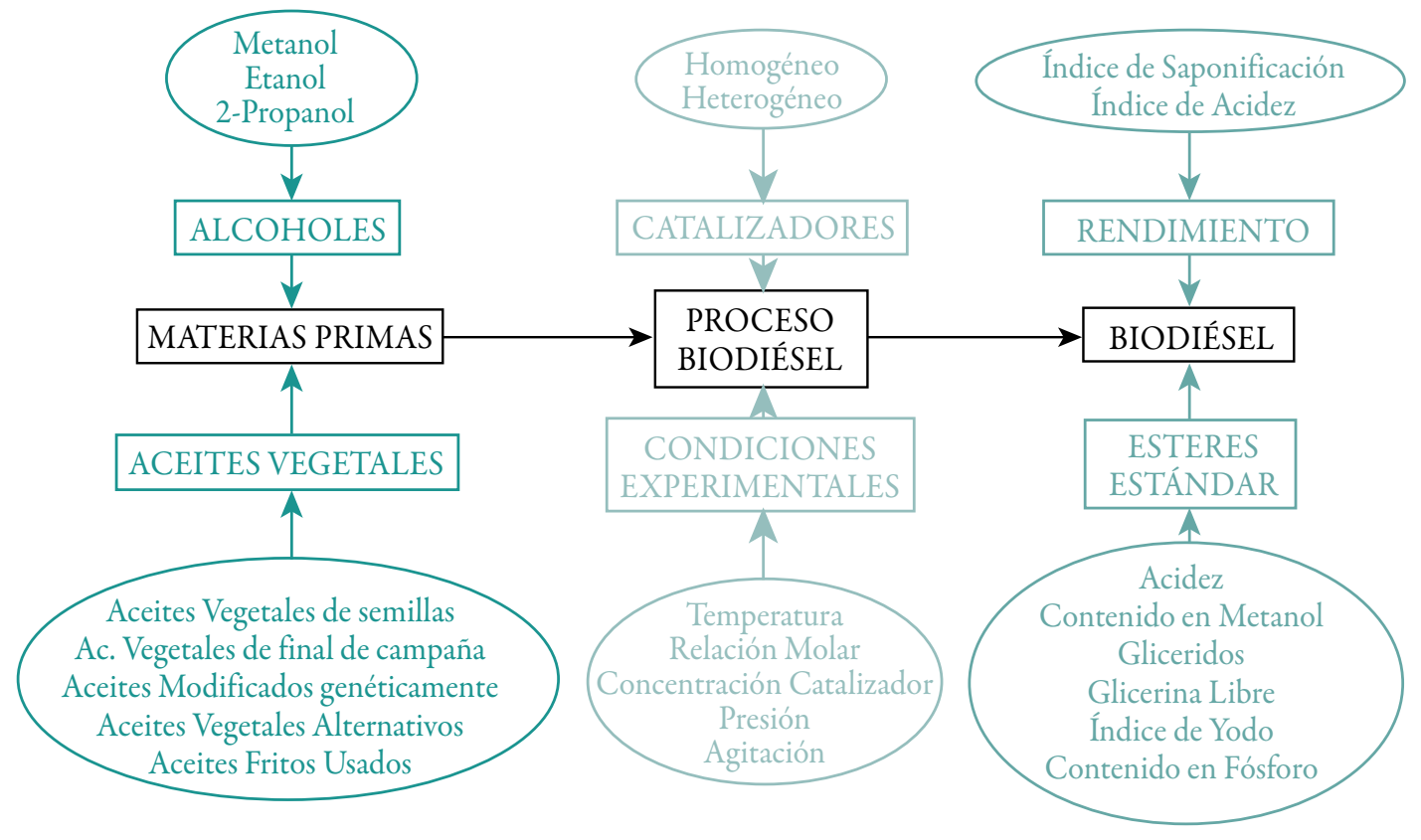




\section{Métodos}

- Valoración del pH de los aceites usados.

- Calentamiento de aceite usado.

- Preparar el metóxido o etóxido de sodio o de potasio.

- Mezclar los aceites usados y etóxido de sodio o de potasio.

- Sedimentación y retirada de glicerina.

- Lavado y secado.

\section{RESULTADOS}

Los resultados a los cuales queremos llegar con la investigación se encuentran en la norma ASTM D 6751-02 (Anexo 1), que describe los métodos y especifica los parámetros de calidad con los que debe cumplir el producto, y se verifica midiendo contenido de agua, existencia de partículas sólidas, residuos de jabón, glicerina, existencia de azufre, etc.

En función de los resultados obtenidos a nivel de laboratorio se desarrollará el prototipo de un reactor Fed Batch que nos permita obtener una máxima producción de éster, con control de temperatura y flujo de alimentación óptimo del catalizador y del alcohol.

\section{REFERENCIAS BIBLIOGRÁFICAS}

1. SPECIFICATION FOR BIODIESEL (B100) - ASTM D6751-06

2. S. D. Romano, E. González Suárez, M. A. Combustibles alternativos. Laborde, Ediciones Cooperativas, 2005.

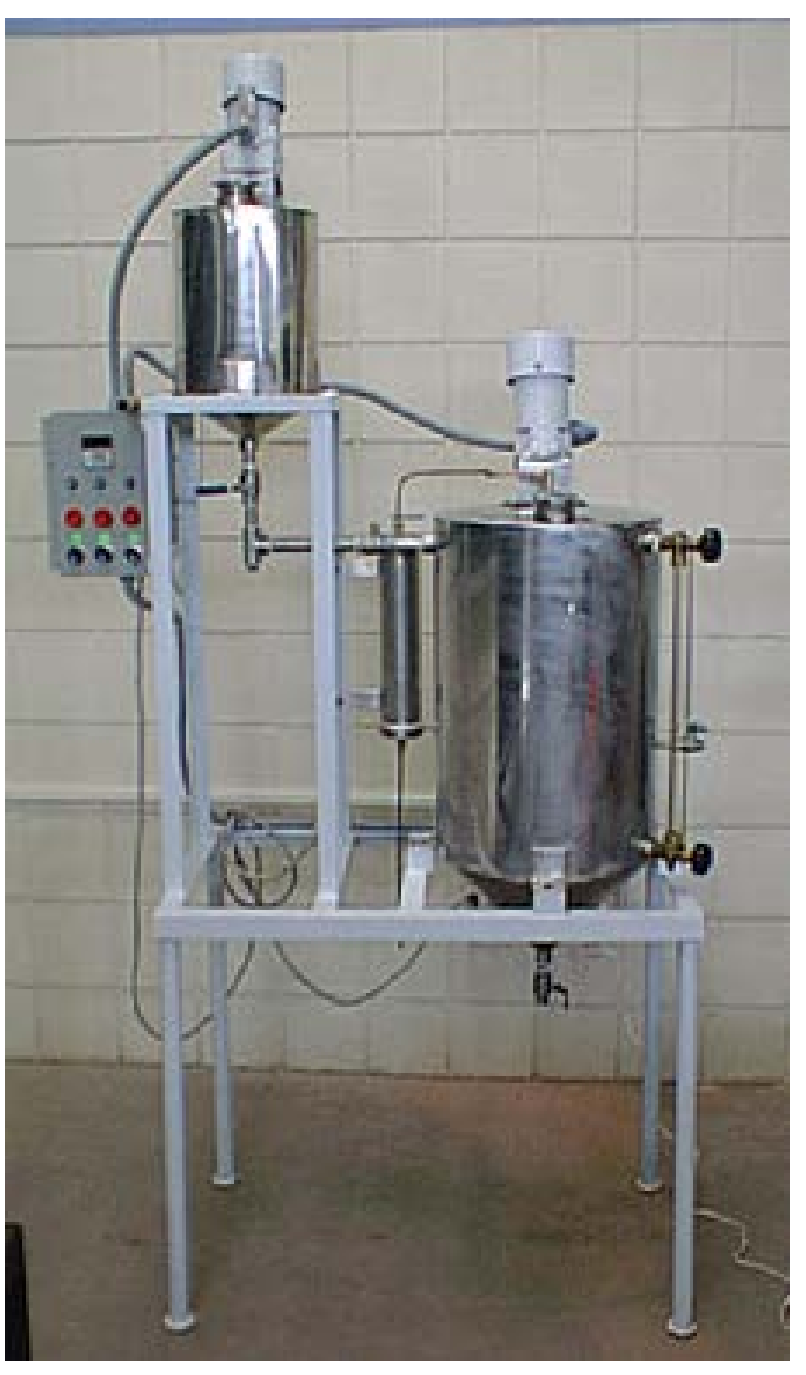

3. ARS -USDA. "Program of Bioenergy and Energy alternatives".

4. Connemann J., Fischer J. Biodiesel in Europe 2000,biodiesel proccesing, technologies and future market development. Alemania, 1999.

5. Mercedes Martínez R. Utilización de aceites usados para la elaboración del biodiesel. Universidad Cumplutense de Madrid.

6. http//www.biodiesel.gov.ar

7. http//www.ebb-eu.org

8. http//www.biodieselverband 
Anexo 1

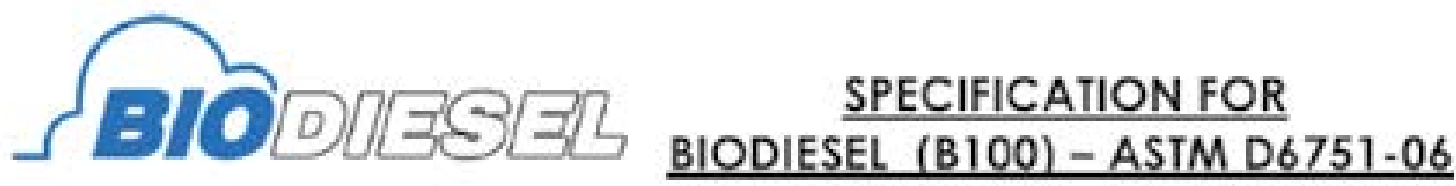

June 2006

Biodiesel is defined as the mono alkyl esters of long chain fatty acids derived from vegelable olls or onimal fats, for use in compression-ignifion (diesel) engines. This specification is for pure (100\%) biodiesel prior to use or blonding with diesel fuel. \#

\begin{tabular}{|c|c|c|c|}
\hline Property & ASTM Method & Limits & Units \\
\hline Flash Point & D93 & $130 \mathrm{~min}$. & Degrees C \\
\hline Water \& Sediment & $\mathrm{D} 2709$ & $0.050 \max$. & $\%$ vol. \\
\hline Kinematic Viscosity, $40 \mathrm{C}$ & D445 & $1.9 \cdot 6.0$ & $\mathrm{~mm}^{2} / \mathrm{sec}$. \\
\hline Sutaled Ash & $\mathrm{DQ7} 4$ & $0.020 \mathrm{max}$. & \% mass \\
\hline $\begin{array}{l}\text { Sulfur } \\
\text { \$ is Grade } \\
\text { \$ s00 Grade }\end{array}$ & D5453 & $\begin{array}{l}15 \mathrm{max} \text {. } \\
500 \mathrm{max} \text {. }\end{array}$ & ppom \\
\hline Copper Strip Cotrosion & DI30 & No. 3 max. & \\
\hline Cetane & D613 & $47 \mathrm{~min}$. & \\
\hline Cloud Point & $\mathrm{D} 2500$ & Report & Degrees C \\
\hline $\begin{array}{l}\text { Carbon Residue } \\
100 \% \text { sample }\end{array}$ & D4530" & 0.050 max. & $\%$ moss \\
\hline Acid Number & D664 & $0.50 \mathrm{max}$ & $\mathrm{mg} \mathrm{KOH} / \mathrm{gm}$ \\
\hline Free Glycerin & D6584 & 0.020 max. & $\%$ moss \\
\hline Tolal Gircerin & D6584 & $0.240 \max$ & 8 moss \\
\hline Phosphorus Content & D 4951 & 0.001 max. & \% moss \\
\hline $\begin{array}{l}\text { Distiliation Temp, } \\
\text { Atmospheric Equivalent } \\
\text { Temperature, } \\
90 \% \text { Recovered }\end{array}$ & 01160 & $360 \max$. & Degrees C \\
\hline Sodum/Potassium & UOP 391 & 5 max, combined & pom \\
\hline
\end{tabular}

